\title{
Cavity ring-down spectroscopy of the fifth overtone of $\mathrm{CO}$
}

\author{
Y. Tan, J. Wang, X.-Q. Zhao, A.-W. Liu, S.-M. Hu \\ Hefei National Laboratory for Physical Sciences at Microscale, iChem center, University \\ of Science and Technology of China, Hefei, 230026 China
}

\begin{abstract}
The extremely weak 6-0 absorption band of the ${ }^{12} \mathrm{C}^{16} \mathrm{O}$ molecule was recorded for the first time using a very sensitive cavity ring-down spectrometer. Frequencies of a few atomic transitions in the $0.8 \mu \mathrm{m}$ region, transferred to the passive modes of a thermally stabilized Fabry-Pérot interferometer, are used to calibrate the observed spectra with an absolute accuracy of $3 \times 10^{-4} \mathrm{~cm}^{-1}$. Line parameters, including line positions, intensities, self-broadening and shifting coefficients were derived by fitting the observed spectra with the Voigt profile.
\end{abstract}

Keywords: cavity ring-down spectroscopy, carbon monoxide, overtone

\section{Introduction}

Carbon monoxide, although being a minor component of the Earth's atmosphere, is the second most abundant neutral molecule in the universe only after $\mathrm{H}_{2}$, but more readily detectable since molecular hydrogen has no dipole transition in its ground state. Carbon monoxide is a main tracer used in the study of astrophysical environments [1,2], and also presents in the atmospheres of Venus [3, 4], Mars [5], and exoplanets [6, 7]. Carbon monoxide plays an important role in the atmospheric carbon cycle on the Earth, produced by biomass burning and fossil fuel consumption [8]. Carbon monoxide is also one of the symptomatic gases used in disease diagnostics based on breath analysis [9].

Email address: smhu@ustc.edu.cn (S.-M. Hu)

Preprint submitted to Elsevier

October 5, 2016

(C) 2016. This manuscript version is made available under the Elsevier user license

http://www.elsevier.com/open-access/userlicense/1.0/ 
Because of its simplicity, the spectrum of CO is a prototype in the study of ro-vibrational spectroscopy, and has been presented in many textbooks. The first rotationally resolved infrared spectroscopic study of $\mathrm{CO}$ was carried out by Lowry in 1924 [10]. High-resolution spectroscopy of the carbon monoxide molecule, including its isotopologues, has been extensively studied, particularly for the relatively strong transitions to low vibrational states. For example, thousands of lines in the fundamental and first overtone bands of ${ }^{12} \mathrm{C}^{16} \mathrm{O},{ }^{13} \mathrm{C}^{16} \mathrm{O},{ }^{12} \mathrm{C}^{18} \mathrm{O}$, and ${ }^{12} \mathrm{C}^{17} \mathrm{O}$ have been analyzed by Farrenq et al. [11]. Here we just give some references of the most recent studies. More references of earlier studies can be found in Refs. [12, 13, 14]. Ngo et al. [15] recently carried out a high-resolution Fourier-transform spectroscopy (FTS) study of the fundamental band and presented the self-broadening and -shifting coefficients. A FTS study of the first overtone 2-0 band of ${ }^{12} \mathrm{C}^{16} \mathrm{O},{ }^{13} \mathrm{C}^{16} \mathrm{O}$ and ${ }^{12} \mathrm{C}^{18} \mathrm{O}$ has been presented by Devi and Benner $[16,17]$. The second overtone 3-0 band has been studied by FTS [18], tunable diode laser spectroscopy (TDLAS) [19, 20], integrated cavity output spectroscopy (ICOS) [21], and cavity ring-down spectroscopy (CRDS) [22]. In general, when the vibrational quantum number increases by one quanta, the overtone band intensity decreases by roughly two orders of magnitude. Consequently, it becomes more difficult to detect higher overtones of CO through absorption methods. Using an absorption cell with an equivalent pathlength $\times$ pressure of $3000 \mathrm{~m} \cdot \mathrm{atm}$, Herzberg and Rao [23] observed the 4-0 band, but failed to detect the 5-0 band. Till now, the 4-0 band is the highest overtone of CO included in the HITRAN [24] database. Recently, Campargue et al. [25] presented CRDS study of the 4-0 band with a frequency accuracy of $5 \times 10^{-4} \mathrm{~cm}^{-1}$. Chung et al. [26] have successfully observed the 5-0 band at $0.96 \mu \mathrm{m}$ using a CRDS instrument based on a Raman-shifted pulsed dye laser, which limited the frequency precision to about $0.1 \mathrm{~cm}^{-1}$. To the best of our knowledge, it is the highest reported overtone of $\mathrm{CO}$ observed so far by absorption.

Here we present the study of the extremely weak 6-0 band of carbon monoxide for the first time. These data could be used to refine the potential energy surface of this important diatomic molecule, to update the spectroscopic database, and also probably in the future study of carbon monoxide in interstellar media. 


\section{Experimental details}

The ro-vibrational lines in the 6 - 0 band of ${ }^{12} \mathrm{C}^{16} \mathrm{O}$ were recorded by a continuous-wave cavity ring-down spectrometer based on a Ti:Sapphire laser (Coherent MBR-110). The details of the spectrometer have been presented elsewhhere [27, 28, 29], and the experimental configuration is similar to that used in our previous studies of the overtone transitions of $\mathrm{H}_{2}$ [30] and $\mathrm{CO}_{2}$ [31]. The ring-down cavity is about $1 \mathrm{~m}$ long and sealed with conflat flanges. A pair of high-reflectivity (HR) mirrors $(R=99.995 \%)$ are used and the mirrors can be adjusted through a set of step-motors by a controller outside the chamber. The ring-down signal is detected by a photo-detector and recorded by an analog-digital converter (ADLink). A nonlinear leastsquare fitting program is applied to fit the data using an exponential-decay function to derive the ring-down time $\tau$. The sample absorption coefficient $\alpha$ at the frequency $\nu$ is derived according to the equation:

$$
\alpha(\nu)=\frac{1}{c \tau(\nu)}-\frac{1}{c \tau_{0}}
$$

where $c$ is the speed of light and $\tau_{0}$ is the ring-down time of the empty cavity. In present study, the typical $\tau_{0}$ value is about $87 \mu$ s and the noise-equivalent absorption coefficient is about $1 \times 10^{-10} \mathrm{~cm}^{-1}$.

The spectrum is calibrated using the passive modes of a Fabry-Pérot interferometer (FPI) made of ultra-low-expansion glass (ULE). The 10-cm-long ULE-FPI (ATFilms Inc.) is thermo-stabilized $(\delta T \sim 1 \mathrm{mK})$ and installed in a vacuum chamber. Atomic lines of Rb at $780 \mathrm{~nm}$ [32] and $795 \mathrm{~nm}$ [33], and of Cs at $852 \mathrm{~nm}$ [34], are used as absolute frequency standards. The frequencies of the longitudinal modes of the ULE-FPI are used to calibrate the recorded spectra with an uncertainty better than $10 \mathrm{MHz}$ in the range of 760-850 nm where the group-delay-dispersion of the interferometer is negligible $[29,35]$.

The spectra of $32 \mathrm{CO}$ lines near $802 \mathrm{~nm}$ were recorded at room temperature $(296 \pm 1 \mathrm{~K})$, using pure carbon monoxide sample (natural isotopic abundance) with stated purity higher than 99.99\%. Different sample pressures in the range of $0.2-0.8 \mathrm{~atm}$, measured by a capacitance gauge with a stated accuracy of $0.5 \%$, were used in the experiments. An example of the spectra, the $\mathrm{R}(5)$ line at $12482.4237 \mathrm{~cm}^{-1}$ is presented in Fig. 1(a). 


\section{Results and discussion}

Parameters of each line were retrieved from fitting the spectra recorded at different pressures using the Voigt profile. We should address that the collisional effect is exceptionally pronounced in CO. As a result, the Voigt profile cannot reproduce the spectra precisely [17]. Because the $6-0$ band is very weak, the signal-to-noise ratio of the recorded spectrum is not sufficient for a non-Voigt analysis. As shown in Fig. 1(b), the fitting residuals of the $\mathrm{R}(5)$ line recorded at different pressures, no obvious deviation from the Voigt profile could be detected so far in this work. For each spectrum, three line parameters were directly derived from the fitting: the line position, the integrated absorption coefficient, and the Lorentzian width (half width at half maximum, HWHM). The Gaussian width was fixed at the calculated value of the Doppler width. Fig. 2 shows the frequency shifts and the integrated absorption coefficients of the $\mathrm{R}(5)$ and $\mathrm{R}(10)$ lines obtained at different sample pressures. Using a linear fit, we derived the line position at the zero pressure limit $\nu_{0}$, the self-pressure induced shift coefficient $\delta$, the line intensity $k$, and the self pressure-broadening coefficient $\gamma$ (not shown in the figure). The results from 32 lines are collected in Table 1.

The precision of the line positions given in Table 1 was examined by the combination differences between the transitions reaching the same upper level. The experimental transition frequency difference between lines $\mathrm{R}(J-1)$ and $\mathrm{P}(J+1)$ and the difference between the ground state energies $E_{J-1}$ and $E_{J+1}$ are shown in Table 2 . Ground state energies are from Ref. [36], with uncertainties at the $10^{-6} \mathrm{~cm}^{-1}$ level. Most of the deviations are below $0.0005 \mathrm{~cm}^{-1}$, being consistent with the experimental accuracy of the measurements.

The ro-vibrational energies in the $V=6$ state of ${ }^{12} \mathrm{C}^{16} \mathrm{O}$ are derived from the transition frequencies given in Table 1 and the ground state rotational energies [36]. Ro-vibrational parameters are obtained by fitting the energies according to the following equation:

$$
E_{v}(J)=G_{v}+B_{v} J(J+1)-D_{v} J^{2}(J+1)^{2}+H_{v} J^{3}(J+1)^{3}
$$

where $G_{v}$ is the vibrational energy, $B_{v}, D_{v}$ and $H_{v}$ are the rotational constant and centrifugal parameters, respectively. The derived parameters are given in Table 3.

The difference between the upper state energies derived from experimental measurements and those from calculated results are plotted in Fig. 3. 
Table 1: Line parameters of the observed transitions of the $6-0$ band of ${ }^{12} \mathrm{C}^{16} \mathrm{O}$. The statistical uncertainties given in parentheses are in unit of the last quoted digit.

\begin{tabular}{|l|llll|}
\hline Line & $\nu_{0}$ & Intensity & $\delta$ & $\gamma$ \\
& $\mathrm{cm}^{-1}$ & $(\mathrm{a})$ & $(\mathrm{b})$ & $(\mathrm{c})$ \\
\hline $\mathrm{R}(11)$ & $12493.48737(8)$ & $5.76(5)$ & $-1.33(2)$ & $6.19(7)$ \\
$\mathrm{R}(10)$ & $12492.17167(22)$ & $6.88(9)$ & $-1.38(4)$ & $6.38(6)$ \\
$\mathrm{R}(9)$ & $12490.64477(7)$ & $7.52(11)$ & $-1.37(2)$ & $6.36(10)$ \\
$\mathrm{R}(8)$ & $12488.90708(11)$ & $8.59(15)$ & $-1.41(2)$ & $6.65(10)$ \\
$\mathrm{R}(7)$ & $12486.95620(14)$ & $8.91(10)$ & $-1.29(3)$ & $6.67(7)$ \\
$\mathrm{R}(6)$ & $12484.79525(11)$ & $8.53(16)$ & $-1.23(3)$ & $6.81(13)$ \\
$\mathrm{R}(5)$ & $12482.42297(18)$ & $9.26(13)$ & $-1.07(4)$ & $7.08(5)$ \\
$\mathrm{R}(4)$ & $12479.84188(8)$ & $8.82(43)$ & $-1.31(2)$ & $7.46(12)$ \\
$\mathrm{R}(3)$ & $12477.04767(136)$ & $7.25(25)$ & $-1.31(30)$ & $7.36(13)$ \\
$\mathrm{R}(2)$ & $12474.04292(11)$ & $6.28(11)$ & $-1.04(2)$ & $8.05(8)$ \\
$\mathrm{R}(1)$ & $12470.82668(77)$ & $4.97(16)$ & $-0.80(11)$ & $8.57(10)$ \\
$\mathrm{R}(0)$ & $12467.40281(128)$ & $2.10(4)$ & $-0.52(22)$ & $8.48(10)$ \\
$\mathrm{P}(1)$ & $12459.92234(50)$ & $2.15(7)$ & $-0.54(9)$ & $8.60(26)$ \\
$\mathrm{P}(2)$ & $12455.87018(184)$ & $4.98(24)$ & $-1.15(32)$ & $8.41(24)$ \\
$\mathrm{P}(3)$ & $12451.60260(39)$ & $6.78(9)$ & $-0.83(10)$ & $7.98(7)$ \\
$\mathrm{P}(4)$ & $12447.12959(12)$ & $8.09(8)$ & $-1.08(2)$ & $7.33(13)$ \\
$\mathrm{P}(5)$ & $12442.44609(7)$ & $9.18(6)$ & $-1.16(2)$ & $7.21(8)$ \\
$\mathrm{P}(6)$ & $12437.55379(12)$ & $10.75(14)$ & $-1.25(2)$ & $7.17(12)$ \\
$\mathrm{P}(7)$ & $12432.45119(48)$ & $10.99(11)$ & $-1.23(9)$ & $6.76(3)$ \\
$\mathrm{P}(8)$ & $12427.14063(11)$ & $10.84(7)$ & $-1.31(3)$ & $6.87(7)$ \\
$\mathrm{P}(9)$ & $12421.62089(9)$ & $10.69(14)$ & $-1.29(2)$ & $6.64(10)$ \\
$\mathrm{P}(10)$ & $12415.89252(33)$ & $10.41(7)$ & $-1.31(7)$ & $6.67(4)$ \\
$\mathrm{P}(11)$ & $12409.95568(22)$ & $9.33(7)$ & $-1.31(5)$ & $6.55(6)$ \\
$\mathrm{P}(12)$ & $12403.81057(5)$ & $8.24(16)$ & $-1.43(1)$ & $6.48(10)$ \\
$\mathrm{P}(13)$ & $12397.45642(25)$ & $7.12(6)$ & $-1.38(4)$ & $6.33(5)$ \\
$\mathrm{P}(14)$ & $12390.89545(15)$ & $6.16(4)$ & $-1.41(4)$ & $6.31(4)$ \\
$\mathrm{P}(15)$ & $12384.12585(25)$ & $5.23(7)$ & $-1.52(4)$ & $6.35(6)$ \\
$\mathrm{P}(16)$ & $12377.14722(2)$ & $4.19(5)$ & $-1.27(1)$ & $6.23(4)$ \\
$\mathrm{P}(17)$ & $12369.96210(22)$ & $3.20(6)$ & $-1.21(4)$ & $5.91(8)$ \\
$\mathrm{P}(18)$ & $12362.57163(22)$ & $2.36(6)$ & $-1.55(3)$ & $5.65(12)$ \\
$\mathrm{P}(19)$ & $12354.97224(84)$ & $1.65(11)$ & $-1.79(10)$ & $5.44(36)$ \\
$\mathrm{P}(20)$ & $12347.16573(81)$ & $1.42(2)$ & $-1.70(10)$ & $5.80(7)$ \\
\hline
\end{tabular}

(a) Line intensity in units of $10^{-29} \mathrm{~cm}$ molecule ${ }^{-1}$. (b) Collision-induced line shift coefficient, in $10^{-2} \mathrm{~cm}^{-1} \mathrm{~atm}^{-1}$. (c) Collision-induced line broadening coefficient (half width at half maximum ), in $10^{-2} \mathrm{~cm}^{-1} \mathrm{~atm}^{-1}$. 
Table 2: Ground state combination differences obtained from the observed transitions in the 6-0 band of ${ }^{12} \mathrm{C}^{16} \mathrm{O}$ (in $\mathrm{cm}^{-1}$ ). Ground state energies are from Ref. [36].

\begin{tabular}{|cccr|}
\hline & Obs. & Calc. & Obs.-Calc. \\
$J$ & $\nu_{R(J-1)}-\nu_{P(J+1)}$ & $E_{J+1}-E_{J-1}$ & $\times 10^{3}$ \\
\hline 2 & 19.22408 & 19.22443 & -0.35 \\
3 & 26.91333 & 26.91318 & +0.15 \\
4 & 34.60161 & 34.60089 & +0.72 \\
5 & 42.28809 & 42.28729 & +0.80 \\
6 & 49.97178 & 49.97207 & -0.29 \\
7 & 57.65462 & 57.65494 & -0.32 \\
8 & 65.33531 & 65.33560 & -0.29 \\
9 & 73.01456 & 73.01377 & +0.79 \\
10 & 80.68909 & 80.68915 & -0.06 \\
11 & 88.36110 & 88.36145 & -0.35 \\
12 & 96.03095 & 96.03037 & +0.58 \\
\hline
\end{tabular}

As shown in the figure, the experimental results agree with that from Ref. [38] within the experimental uncertainty. Mishra et al. [37] has obtained the parameters of the $V=6$ upper state using the line positions got from Fourier-transform emission spectroscopy. As shown in Table. 3, by this work, the accuracy of the band center has been improved by about two orders of magnitude. In the same table, we also include the ro-vibrational parameters derived from the Dunham coefficients given in Refs. [11, 12, 14], which have been obtained from a "global fitting" of all the transitions to different vibrational states of CO. These parameters have also been applied to derive the line positions and the rms deviations from the experimental results obtained in this work are also given in the same table. The line positions from the parameters from Mishra et al. have a deviation of $-0.0049(18) \mathrm{cm}^{-1}$, and those from Refs. $[11,12,14]$ all have a deviation of $+0.0003(8) \mathrm{cm}^{-1}$. The rms values from Refs. $[11,12,14]$ are all $0.00078 \mathrm{~cm}^{-1}$, slightly larger than the value from the parameters given in this work.

The line intensities together with the statistical uncertainties from the measurements are also given in Table 1 . The relative statistical uncertainties are about $1-5 \%$ for most lines. A systematic uncertainty of about $2 \%$ needs to be considered, which comes from the line profile model applied in the spectral fit. Fig. 4 shows the comparison of the line intensities obtained from the present study and that from a recent semi-empirical dipole moment 
Table 3: Spectroscopic constants (in $\mathrm{cm}^{-1}$ ) for the vibrational states of ${ }^{12} \mathrm{C}^{16} \mathrm{O}$. Values given in parentheses are the uncertainties in last quoted digit. Terms of higher orders are neglected.

\begin{tabular}{|llllll|}
\hline$G_{v}$ & $B_{v}$ & $D_{v} \times 10^{6}$ & $H_{v} \times 10^{12}$ & $r m s \times 10^{3}$ & Ref. \\
\hline 0.0 & $1.92252895231(93)$ & $6.1210643(53)$ & $5.7266(33)$ & 0.00043 & {$[36]$} \\
\hline $12463.765(15)$ & $1.8175154(53)$ & $6.1125(48)$ & $3.9(11)$ & 1.84 & {$[37]$} \\
12463.768596 & 1.817533272 & 6.12222207 & 4.904510 & 0.78 & {$[11]$} \\
12463.768582 & 1.817533336 & 6.12225340 & 4.909270 & 0.78 & {$[12]$} \\
12463.768565 & 1.817533298 & 6.12225378 & 4.912309 & 0.78 & {$[14]$} \\
$12463.76825(16)$ & $1.8175321(27)$ & $6.1129(90)$ & $5.7266^{a}$ & 0.33 & this \\
\hline
\end{tabular}

${ }^{a}$ Value fixed at the value of ground state.

function (DMF) [38]. As stated in Ref. [38], previous ab initio DMF [39] and semi-empirical DMF [40] considerably overestimated the 6-0 band strength by three orders of magnitude. The line intensities from the recent $a b$ initio DMF [38] are still about over 30\% larger than the experimental results obtained in this work. A part of our results, including $R(5), R(7), R(9), R(10)$ (triangles on Fig 4), have been used to construct a semi-empirical DMF [38]. In this way, the semi-empirical DMF results agree very well with the experimental results. As shown in the lower panel of Fig. 4, for most of the unblended lines, the relative deviation is about 1-5\%, being consistent to the experimental statistical uncertainties given in Table 1.

Accurate line profile parameters of the $\mathrm{CO}$ molecule are also of great needs in studies of planetary atmospheres. By fitting the profiles of absorption lines observed at different sample pressures, we derived the Lorentzian widths and positions of each line using the conventional Voigt profile. Linear fit of these results yield the coefficients of self pressure-induced line broadening and shift. The self-broadening coefficients determined from this work along with previous studies are shown in Fig. 5a. The values derived from the 6-0 band are consistent with those from other bands including the fundamental band. We cannot find any clear evidence of vibrational dependence. The pressure-induced line shifts are shown in Fig. 5b. The coefficients for the 1-0, 2-0 and 3-0 bands have been investigated by Ngo et al. [15]. Here we use the approach given in Ref. [15] to convert the values from other bands into values for the 6-0 band. It has been shown by Hartmann that the line shift of linear molecule like $\mathrm{CO}$ consists of symmetric and antisymmetric parts with 
respect to the rotational quantum number $m(m$ is $-J$ in the $\mathrm{P}$ branch and $J+1$ in the $\mathrm{R}$ branch). [41] The pressure shift in the $v_{1}^{\prime}-v_{1}^{\prime \prime}$ band is related to that in the $v_{0}-v_{0}^{\prime \prime}$ band through the following equation:

$\delta\left(v_{1}^{\prime} \leftarrow v_{1}^{\prime \prime}, m\right)=\delta\left(v_{0}^{\prime} \leftarrow v_{0}^{\prime \prime}, m\right)+\left(v_{1}^{\prime}-v_{0}^{\prime}-v_{1}^{\prime \prime}+v_{0}^{\prime \prime}\right)\left[\delta\left(v_{0}^{\prime} \leftarrow v_{0}^{\prime \prime}, m\right)+\delta\left(v_{0}^{\prime} \leftarrow v_{0}^{\prime \prime},-m\right)\right] / 2$

The obtained results are shown in Fig. 5b. The coefficients derived from different bands show a similar trend as a function of $m$, but with a systematic deviation among different bands. It could be a result of different line profiles applied in spectral analysis and different experimental conditions used in the measurements.

\section{Conclusion}

We present the first absorption spectroscopic analysis of the extremely weak 5 th overtone band of monoxide carbon near $804 \mathrm{~nm}$. Parameters of the lines in the 6-0 band of ${ }^{12} \mathrm{C}^{16} \mathrm{O}$ have been derived from the observed spectra. Line positions have been determined with an accuracy of $3 \times 10^{-4} \mathrm{~cm}^{-1}$, line intensities, self-pressure broadening and shift coefficients are determined with a relative uncertainty of about $5 \%$. The line parameters can be used to constrain the effective ro-vibrational coefficients of the $\mathrm{CO}$ molecule.

\section{Acknowledgements}

We are indebt to Dr. I. E. Gordon (Harvard-Smithsonian Center for Astrophysics) and Dr. S. Tashkun (Zuev Institute of Atmospheric Optics) for helpful discussions. This work is jointly supported by NBRPC (2013CB834602), by NSFC (21411130183, 21225314, 21303176, 21427804), and by CAS (XDB21020100).

[1] Visser R, van Dishoeck EF, Black JH. The photodissociation and chemistry of CO isotopologues: applications to interstellar clouds and circumstellar disks. Astron Astrophys 2009; 503: 323-356.

[2] Dame TM, Hartmann D, Thaddeus P. The Milky Way in molecular clouds: A new complete CO survey. Astrophys J 2001; 547: 792-813.

[3] Connes P, Connes J, Kaplan LD, Benedict WS. Carbon monoxide in venus atmosphere. Astrophys J 1968; 152: 731. 
[4] Bezard B, Debergh C, Crisp D, Maillard JP. The deep atmosphere of venus revealed by high-resolution nightside spectra. Nature 1990; 345: $508-511$.

[5] Owen T, Biemann K, Rushneck DR, Biller JE, Howarth DW, Lafleur AL. The composition of the atmosphere at the surface of mars. J Geophys Res 1977; 82: 4635-9.

[6] Brogi M, de Kok RJ, Birkby JL, Schwarz H, Snellen IAG. Carbon monoxide and water vapor in the atmosphere of the non-transiting exoplanet HD 179949 b. Astron Astrophys 2014; 565: A124.

[7] Konopacky QM, Barman TS, Macintosh BA, Marois C. Detection of carbon monoxide and water absorption lines in an exoplanet atmosphere. Science 2013; 339: 1398-1401.

[8] Vidot J, Landgraf J, Hasekamp OP, Butz A, Galli A, Tol P, Aben I. Carbon monoxide from shortwave infrared reflectance measurements: A new retrieval approach for clear sky and partially cloudy atmospheres. Remote Sens Environ 2012; 120: 255-266.

[9] Wang C, Sahay P. Breath analysis using laser spectroscopic techniques: Breath biomarkers, spectral fingerprints, and detection limits. Sensors 2009; 9: 8230-8262.

[10] Lowry EF. The infrared absorption spectrum of carbon monoxide. J Opt Soc Amer \& Rev Sci Instrum 1924; 8: 647-658.

[11] Farrenq R, Guelachvili G, Sauval AJ, Grevesse N, Farmer CB. Improved dunham coefficients for $\mathrm{CO}$ from infrared solar lines of high rotational excitation. J Mol Spectrosc 1991; 149: 375-390.

[12] Coxon JA, Hajigeorgiou PG. Direct potential fit analysis of the $X^{1} \Sigma^{+}$ ground state of CO. J Chem Phys 2004; 121: 2992-3008.

[13] Tashkun SA, Velichko TI, Mikhailenko SN. Critical evaluation of measured pure-rotation and rotation-vibration line positions and an experimental dataset of energy levels of ${ }^{12} \mathrm{C}^{16} \mathrm{O}$ in $\mathrm{x}^{1} \Sigma^{+}$state. J Quant Spectrosc Radiat Transf 2010; 111: 1106-1116. 
[14] Velichko TI, Mikhailenko SN, Tashkun SA. Global Multi-isotopologue fit of measured rotation and vibrationCrotation line positions of $\mathrm{CO}$ in $X^{1} \Sigma^{+}$state and new set of mass-independent Dunham coefficients. J Quant Spectrosc Radiat Transf 2012; 113: 1643-1655.

[15] Ngo NH, Landsheere X, Pangui E, Morales SB, Tran H, Hartmann JM. Self-broadening and -shifting of very intense lines of the $1 \leftarrow 0$ band of ${ }^{12} \mathrm{C}^{16} \mathrm{O}$. J Quant Spectrosc Radiat Transf 2014; 149: 285-290.

[16] Devi VM, Benner DC, Smith MAH, Mantz AW, Sung K, Brown LR. Spectral line parameters including temperature dependences of airbroadening for the $2 \leftarrow 0$ bands of ${ }^{13} \mathrm{C}^{16} \mathrm{O}$ and ${ }^{12} \mathrm{C}^{18} \mathrm{O}$ at $2.3 \mu \mathrm{m}$. J Mol Spectrosc 2012; 276: 33-48.

[17] Devi VM, Benner DC, Smith MAH, Mantz AW, Sung K, Brown LR, Predoi-Cross A. Spectral line parameters including temperature dependences of self- and air-broadening in the $2 \leftarrow 0$ band of $\mathrm{CO}$ at $2.3 \mu \mathrm{m}$. J Quant Spectrosc Radiat Transf 2012; 113: 1013-1033.

[18] Picqué N, Guelachvili G, Dana V, Mandin JY. Absolute line intensities, vibrational transition moment, and self-broadening coefficients for the 3-0 band of ${ }^{12} \mathrm{C}^{16} \mathrm{O}$. J Mol Spectrosc 2000; 517: 427-434.

[19] Henningsen J, Simonsen H, Mogelberg T, Trudso E. The $0 \rightarrow 3$ overtone band of CO: Precise linestrengths and broadening parameters. J Mol Spectrosc 1999; 193: 354-362.

[20] Swann WC, Gilbert SL. Pressure-induced shift and broadening of 1560$1630 \mathrm{~nm}$ carbon monoxide wavelength-calibration lines. J Opt Soc Am B 2002; 19: 2461-2467.

[21] Engel GS, Drisdell WS, Keutsch FN, Moyer EJ, Anderson JG. Ultrasensitive near-infrared integrated cavity output spectroscopy technique for detection of $\mathrm{CO}$ at $1.57 \mu \mathrm{m}$ : new sensitivity limits for absorption measurements in passive optical cavities. Appl Opt 2006; 45: 9221-9229.

[22] Mondelain D, Sala T, Kassi S, Romanini D, Marangoni M, Campargue $\mathrm{A}$. Broadband and highly sensitive comb-assisted cavity ring down spectroscopy of $\mathrm{CO}$ near $1.57 \mu \mathrm{m}$ with sub-mHz frequency accuracy. J Quant Spectrosc Radiat Transf 2015; 154: 35-43. 
[23] Herzberg G, Rao KN. Rotation-vibration spectra of diatomic and simple polyatomic molecules with long absorbing paths. 2. the spectrum of carbon monoxide below 1.2 $\mu$ J Chem Phys 1949; 17: 1099-1102.

[24] Rothman LS, Gordon IE, Babikov Y, Barbe A, Chris Benner D, Bernath PF, et al. The HITRAN2012 molecular spectroscopic database. J Quant Spectrosc Radiat Transf 2013; 130: 4-50.

[25] Campargue A, Karlovets EV, Kassi S. The 4-0 band of carbon monoxide by high sensitivity cavity ring down spectroscopy near $8200 \mathrm{~cm}^{-1}$. J Quant Spectrosc Radiat Transf 2015; 154: 113-119.

[26] Chung CY, Ogilvie JF, Lee YP. Detection of vibration-rotational band $5-0$ of ${ }^{12} \mathrm{C}^{16} \mathrm{O} \mathrm{X}^{1} \Sigma^{+}$with cavity ringdown absorption near $0.96 \mu \mathrm{m}$. J Phys Chem A 2005; 109: 7854-7858.

[27] Gao B, Jiang W, Liu AW, Lu Y, Cheng CF, Cheng GS, Hu SM. Ultrasensitive near-infrared cavity ring-down spectrometer for precise line profile measurement. Rev Sci Instrum 2010; 81: 043105.

[28] Pan H, Cheng CF, Sun YR, Gao B, Liu AW, Hu SM. Laser-locked, continuously tunable high resolution cavity ring-down spectrometer. Rev Sci Instrum 2011; 82: 103110.

[29] Cheng CF, Sun YR, Pan H, Lu Y, Li XF, Wang J, Liu AW, Hu, SM. Cavity ring-down spectroscopy of doppler-broadened absorption line with sub-mhz absolute frequency accuracy. Opt Expr 2012; 20: 99569963.

[30] Hu SM, Pan H, Cheng, CF, Sun, YR, Li XF, Wang J, Campargue A, Liu AW. The $v=3 \leftarrow 0 \mathrm{~S}(0)-\mathrm{S}(3)$ electric quadrupole transitions of $\mathrm{H}_{2}$ near $0.8 \mu \mathrm{m}$. Astrophys J 2012; 749: 76.

[31] Lu Y, Liu AW, Li XF, Wang J, Cheng CF, Sun YR, Lambo R, Hu SM. Line parameters of the $782 \mathrm{~nm}$ band of $\mathrm{CO}_{2}$. Astrophys J 2013; 775: 71 .

[32] Ye J, Swartz S, Jungner P, Hall JL. Hyperfine structure and absolute frequency of the ${ }^{87} \mathrm{Rb}{ }^{5} \mathrm{P}_{3 / 2}$ state. Opt Lett 1996; 21: 1280-1282.

[33] Maric M, McFerran JJ, Luiten AN. Frequency-comb spectroscopy of the $D_{1}$ line in laser-cooled rubidium. Phys Rev A 2008; 77: 032502. 
[34] Udem T, Reichert J, Hänsch TW, Kourogi M. Absolute optical frequency measurement of the cesium D2 line. Phys Rev A 2000; 62: 031801.

[35] Tan Y, Wang J, Cheng CF, Zhao XQ, Liu AW, Hu SM. Cavity ringdown spectroscopy of the electric quadrupole transitions of $\mathrm{H}_{2}$ in the 784-852 nm region. J Mol Spectrosc 2014; 300: 60-64.

[36] Winnewisser G, Belov SP, Klaus T, Schieder R. Sub-doppler measurements on the rotational transitions of carbon monoxide. J Mol Spectrosc 1997; 184: 468-472.

[37] Mishra AP, Shetty BJ, Kshirsagar RJ. Fourier transform emission spectroscopy of $\Delta v=2$ sequence bands of the $\mathrm{CO}$ molecule in the ground electronic state. J Mol Spectrosc 2005; 232: 296-307.

[38] Li G, Gordon IE, Rothman LS, Tan Y, Hu SM, Kassi S, Campargue A, Medvedev ES. Rovibrational line lists for nine isotopologues of the co molecule in the $\mathrm{X}^{1} \Sigma^{+}$ground electronic state. Astrophys J Supp Ser 2015; 216: 15 .

[39] Langhoff SR, Bauschlicher CW. Global dipole-moment function for the $\mathrm{X}^{1} \Sigma^{+}$ground-state of CO. J Chem Phys 1995; 102: 5220-5225.

[40] Chackerian C, Tipping RH. Vibration-rotational and rotational intensities for CO isotopes. J Mol Spectrosc 1983; 99: 431-449.

[41] Hartmann JM. A simple empirical model for the collisional spectral shift of air-broadened $\mathrm{CO}_{2}$ lines. J Quant Spectrosc Radiat Transf 2009; 110: 2019-2026. 
Figure 1: (a) The R(5) line recorded at different pressures: $22.5 \mathrm{kPa}, 41.5 \mathrm{kPa}, 60.5 \mathrm{kPa}$ and $81.8 \mathrm{kPa}$; (b) Fitting residuals. Voigt profile was applied in the fitting.

Figure 2: $\mathrm{R}(5)$, and $\mathrm{R}(10)$ lines in the (6-0) band of ${ }^{12} \mathrm{C}^{16} \mathrm{O}$, (a) position shift and (b) integrated absorbance of the spectrum recorded at different sample pressures.

Figure 3: Differences between the upper level energies calculated with parameters given in Table 3 and the experimental line positions and those from the DMS calculated values [38].

Figure 4: Line intensities of the 6-0 band of ${ }^{12} \mathrm{C}^{16} \mathrm{O}$ : solid circles: results from the effective dipole moment function (DMF) in Ref. [38]; open circles: this work; open triangles: part of the experimental data in this work used to constrain the DMF calculation. The lower panel shows the relative ratios of the experimental values over those from the DMF calculation [38]. Note the error bars take into account the statistical uncertainties only.

Figure 5: Pressure-induced line broadening and shifting coefficients of the ro-vibrational transitions of $\mathrm{CO}(m$ is $-J$ in the $\mathrm{P}$ branch and $J+1$ in the $\mathrm{R}$ branch). 


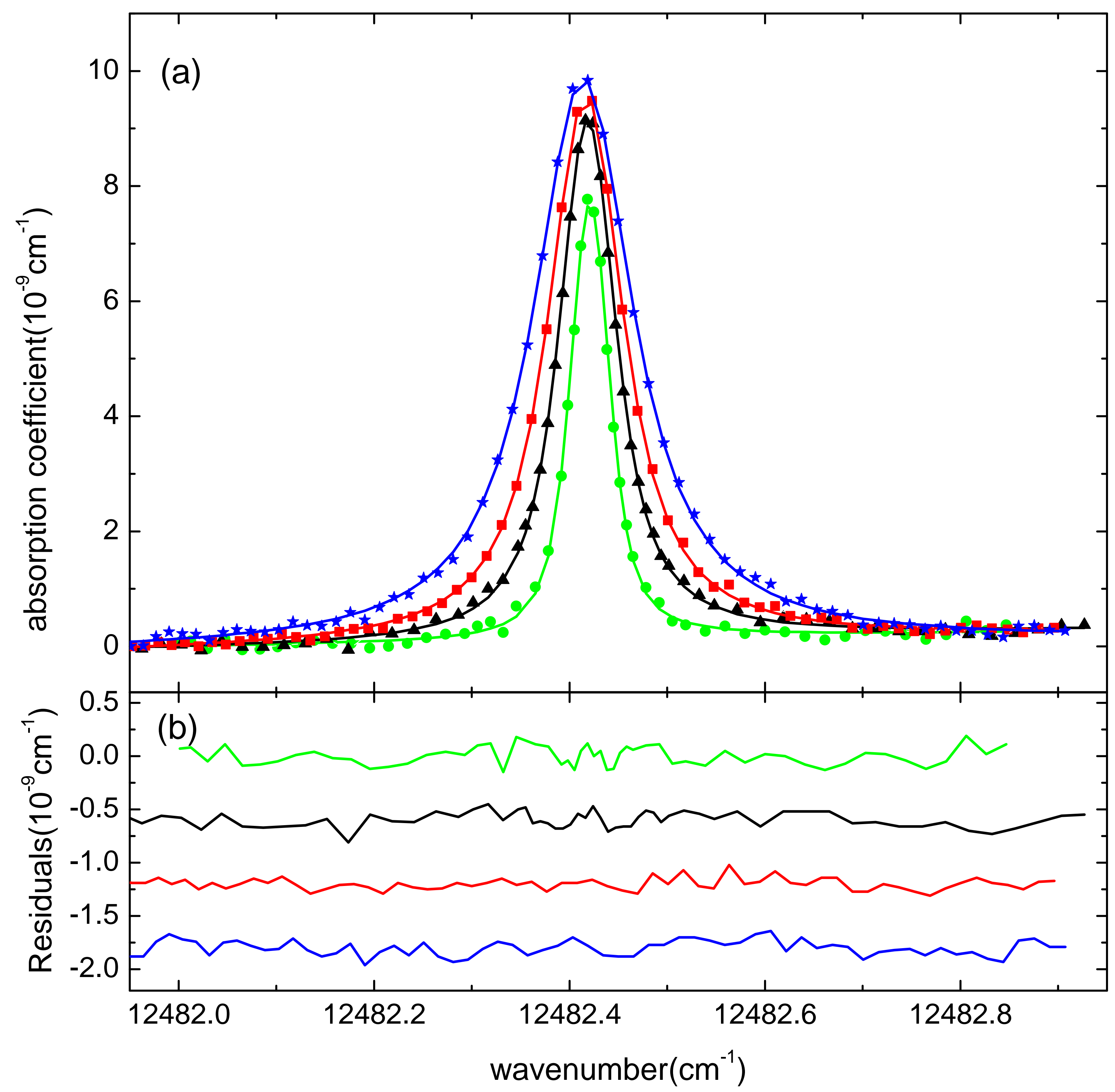




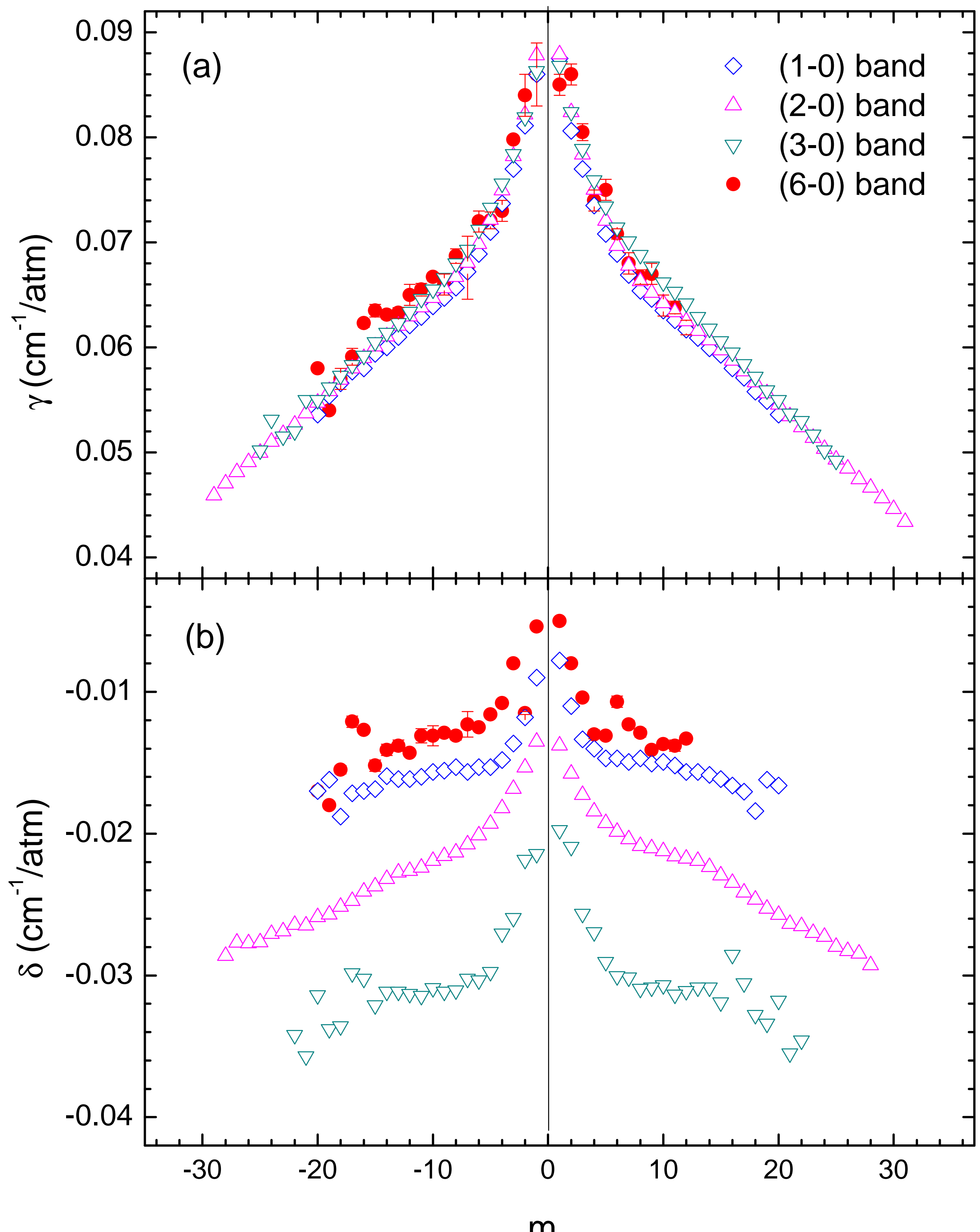

$\mathrm{m}$ 




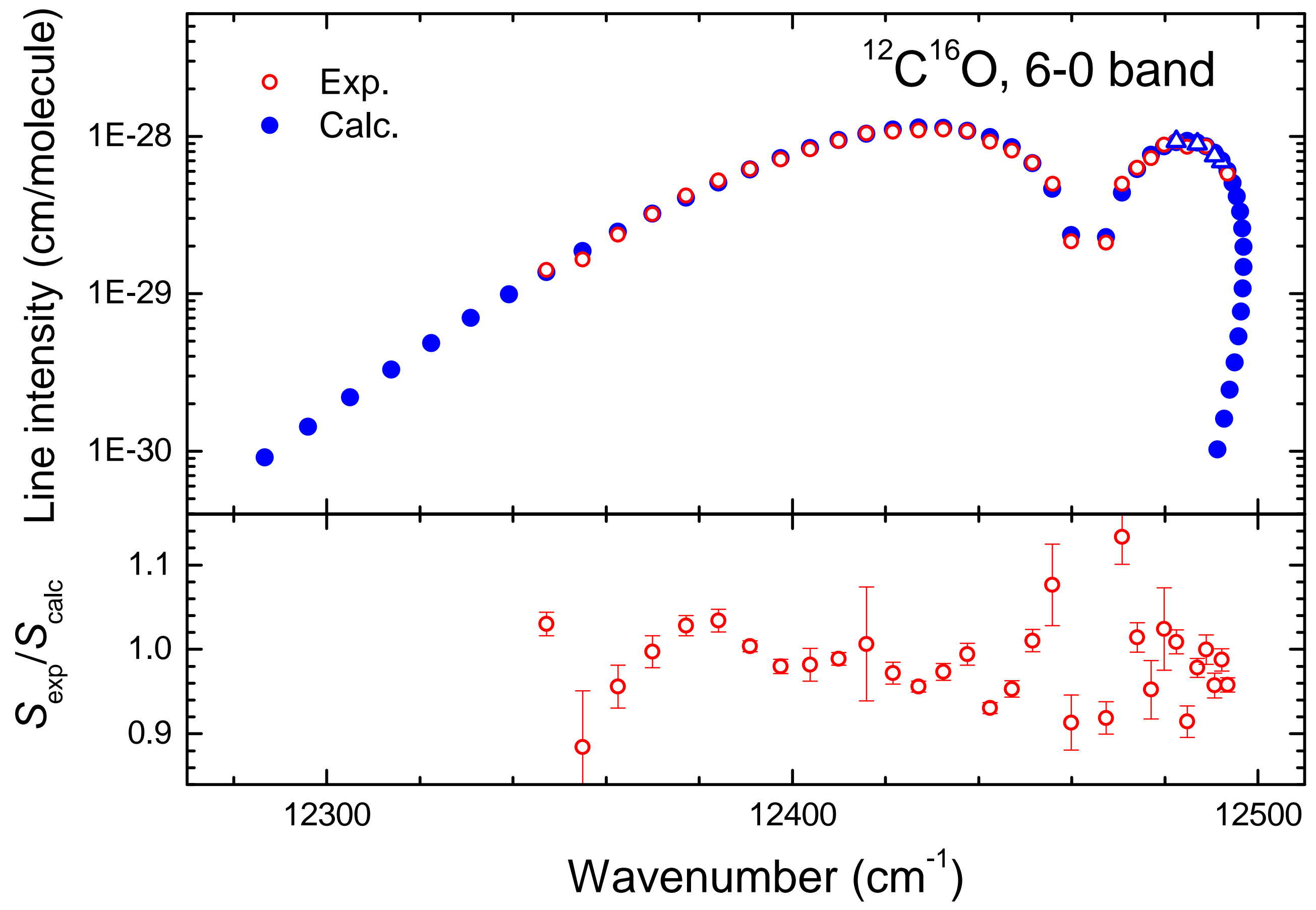




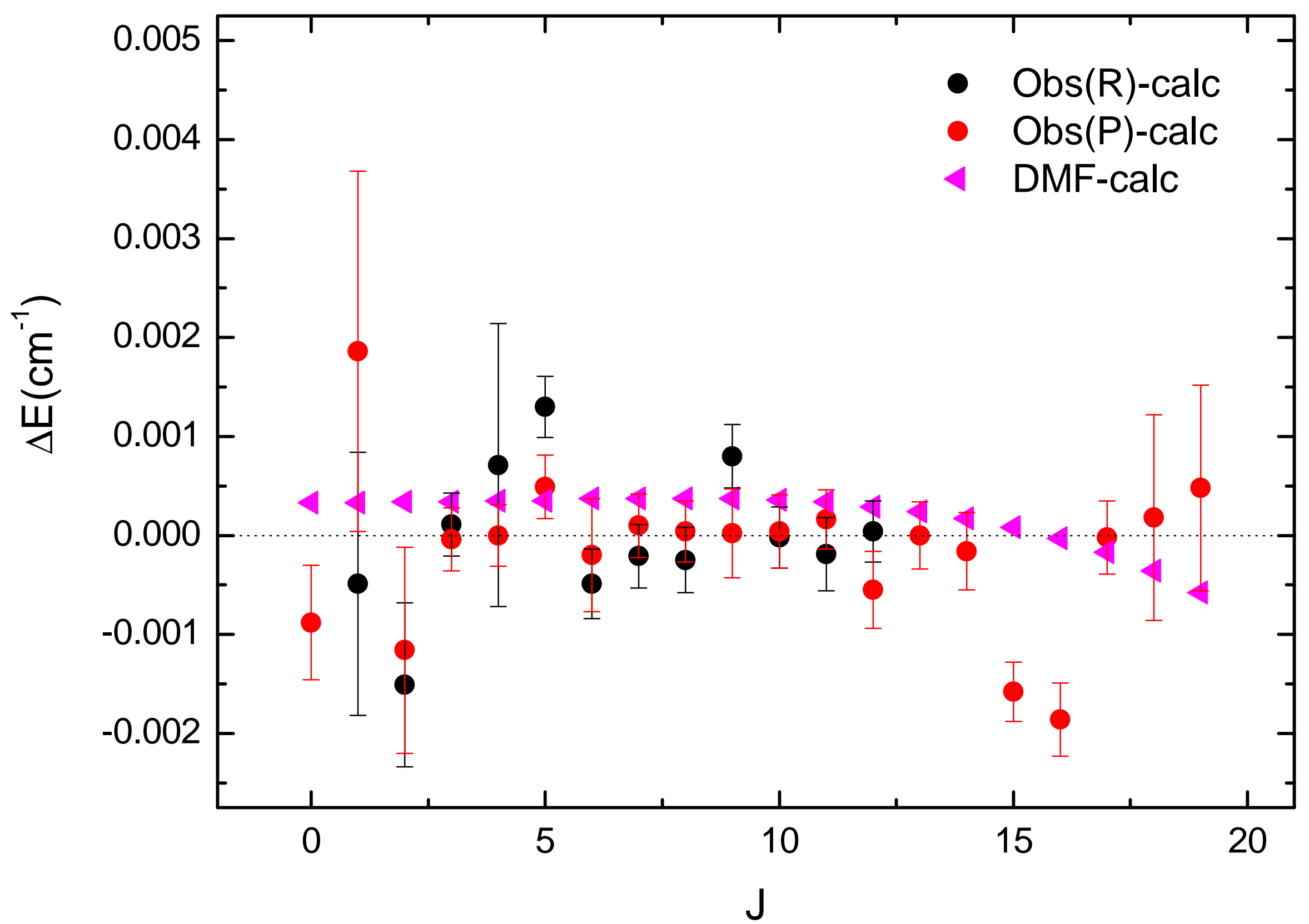

